\title{
A combined variation of the musculocutaneous nerve associated with a supernumerary head of the biceps brachii muscle
}

\author{
S.H. Lee ${ }^{1}$, J.Y. Jeon ${ }^{1}$, S.P. Yoon ${ }^{2}$ \\ ${ }^{1}$ Medical Course, School of Medicine, Jeju National University, Jeju-Do, Republic of Korea \\ ${ }^{2}$ Department of Anatomy, School of Medicine, Jeju National University, Jeju-Do, Republic of Korea
}

[Received 8 January 2014; Accepted 28 January 2014]

\begin{abstract}
Single anatomical variation in the upper limb is common, but the coexistence of neuromuscular anomalies is still rare. We found a combined neuromuscular variation in the upper limb in a 61-year-old Korean male cadaver, whose cause of death was laryngeal carcinoma, during a routine dissection course for medical students. In his left arm, a supernumerary head of the biceps brachii muscle attached to the anteromedial surface of mid-humerus and united distally with the long and short heads of the biceps brachii muscle (BBM). The musculocutaneous nerve, which did not pierce the coracobrachialis muscle but gave 2 muscular branches, had a communicating branch to the median nerve. Since the presence of the supernumerary head of the BBM might affect the course and branching of the musculocutaneous nerve, knowing different patterns of the musculocutaneous variation associated with the BBM variations is essential for anatomists and clinicians. (Folia Morphol 2014; 73, 3: 366-369)
\end{abstract}

Key words: supernumerary head, biceps brachii muscle, musculocutaneous nerve, communicating branch

\section{INTRODUCTION}

Neuromuscular anomalies are common in the upper limbs: the brachial plexus is the most variable part of the peripheral nervous system [6] and the biceps brachii muscle (BBM) is one of the most variable muscles in the human body [2]. Variations in the course and distribution of the musculocutaneous nerve (McN) [5] and the supernumerary head of the BBM [13] have been classified, because the prevalence of each variation reached about $34 \%$ and $22.4 \%$, respectively [7]. Single anatomical variation in the upper limb is common, but the coexistence of neuromuscular anomalies is not common. Kosugi et al. [7] classified the branching patterns of communications from the McN to the median nerve
(MN) with the supernumerary head of the BBM. This report presents a combined variation with a unique course and communicating branch of the $\mathrm{McN}$ and a supernumerary head of the BBM on one arm during routine educational dissection.

\section{CASE REPORT}

During a routine dissection at Jeju National University Medical School in 2013, combined variations of the McN associated with a supernumerary head of the BBM were observed in a 61-year-old Korean male cadaver, whose cause of death was 'laryngeal carcinoma'. The protocol for the current report did not include any specific issue that needed to be approved by the ethics committee of our institution

Address for correspondence: Dr S.P. Yoon, Department of Anatomy, School of Medicine, Jeju National University, 102 Jejudaehak-ro, Jeju-Si, Jeju-Do 690-756, Republic of Korea, tel: +82 64 7543823, fax: +82 64 7252593, e-mail: spyoon@jejunu.ac.kr 


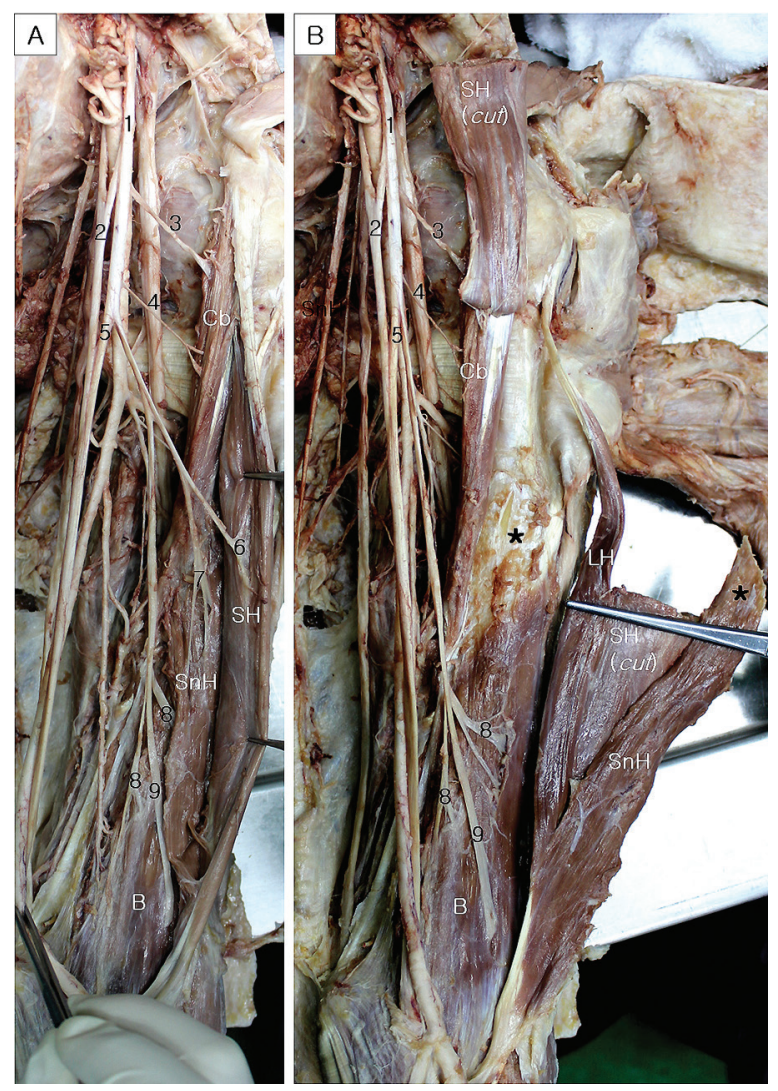

Figure 1. Dissection of the combined neuromuscular variations of the supernumerary head $(\mathrm{SnH})$ of the biceps brachii muscle and a communicating branch of the musculocutaneous nerve before $(\mathbf{A})$ and after (B) reflecting the biceps brachii muscle; 1 - musculocutaneous nerve; 2 - median nerve; 3 - nerve to coracobrachialis; 4 - nerve to coracobrachialis; 5 - communicating branch from the musculocutaneous nerve; 6 - nerve to biceps brachii; 7 nerve to $\mathrm{SnH} ; 8$ - nerve to brachialis; 9 - lateral cutaneous nerve of forearm; Asterisk — proximal attachment of $\mathrm{SnH}$; B — brachialis; $\mathrm{Cb}$ - coracobrachialis; $\mathrm{SH}$ - short head of the biceps brachii; LH — long head of the biceps brachii.

and it conformed to the provisions of the Declaration of Helsinki in 1995. Gross dissection was performed in the customary fashion. Accidental injury on the left upper arm was ruled out as no incision or injury marks were observed on the skin of the arm.

We found that the long head of the BBM was relatively smaller in terms of the normal balance between a long head and a short head of the BBM. So we observed muscles of left arm in detail and in this process we found a supernumerary head of the BBM. The origin of supernumerary head of the BBM was located on antero-medial surface of left humerus between the origin of the brachialis muscle and the insertion of the coracobrachialis muscle (Cb) (Fig. 1A). The long head, the short head and the supernume- rary head of the BBM were united and inserted on the radial tuberosity and fascia of forearm through bicipital aponeurosis.

Although the McN pierced the $\mathrm{Cb}$ on right upper limb, the McN did not penetrate the $\mathrm{Cb}$ on the left upper limb. Instead of not penetrating the $\mathrm{Cb}$, there were 2 branches to $\mathrm{Cb}$ from the McN (Fig. 1B). And then the $\mathrm{McN}$ divided into 2 branches at the upper $1 / 3$ of the humerus, the lateral one was the main $\mathrm{McN}$ and the medial one was the communicating branch from the McN to MN. The main McN gave 2 branches: one was to the BBM and to the supernumerary head of the BBM, the other was to the brachialis muscle and continued to the lateral cutaneous nerve of forearm. The former ran between the short head and the supernumerary head of the BBM and the latter ran between the supernumerary head of the BBM and the brachialis muscle.

\section{DISCUSSION}

As the classification of Guerri-Guttenberg and Ingolotti [5] revealed the most detailed descriptions, we followed the classification for further discussion on the variation of $\mathrm{McN}$. In this case, McN did not pierce the $\mathrm{Cb}$ and had one communicating branch to the $\mathrm{MN}$, which corresponds with the previous classification type 1-B-1 [5]. The supernumerary heads of the BBM were classified according to their origin and location [13]: superior humeral heads (1.4\%), infero-medial humeral heads (6\%), and infero-lateral humeral heads $(0.3 \%)$. According to the previous classification, the present case has an infero-medial humeral head of the BBM.

Communications between $\mathrm{McN}$ and $\mathrm{MN}$ were observed in $48 \%$ [9] or $57.3 \%$ [7] of those cases with the excessive head of the BBM. The combined neuromuscular variations were classified by Kosugi et al. [7] as follows: Group I is a pattern without the communication between the $\mathrm{McN}$ and the MN (42.7\%); Group II has a communicating branch from the $\mathrm{McN}$ to the MN (32\%); Group III has a branch running from the MN to the McN (16\%), Group IV has above 2 communications (6.7\%), and Group V has various other patterns (2.7\%). According to this classification, the present case comes under Group II.

In recently published literatures, only a few cases were presented where variation of the McN coexisted with a supernumerary-headed BBM: one showed the absence of the McN $[2,3]$ and the other had a communicating branch to the $\mathrm{MN}[1,10]$. According to 


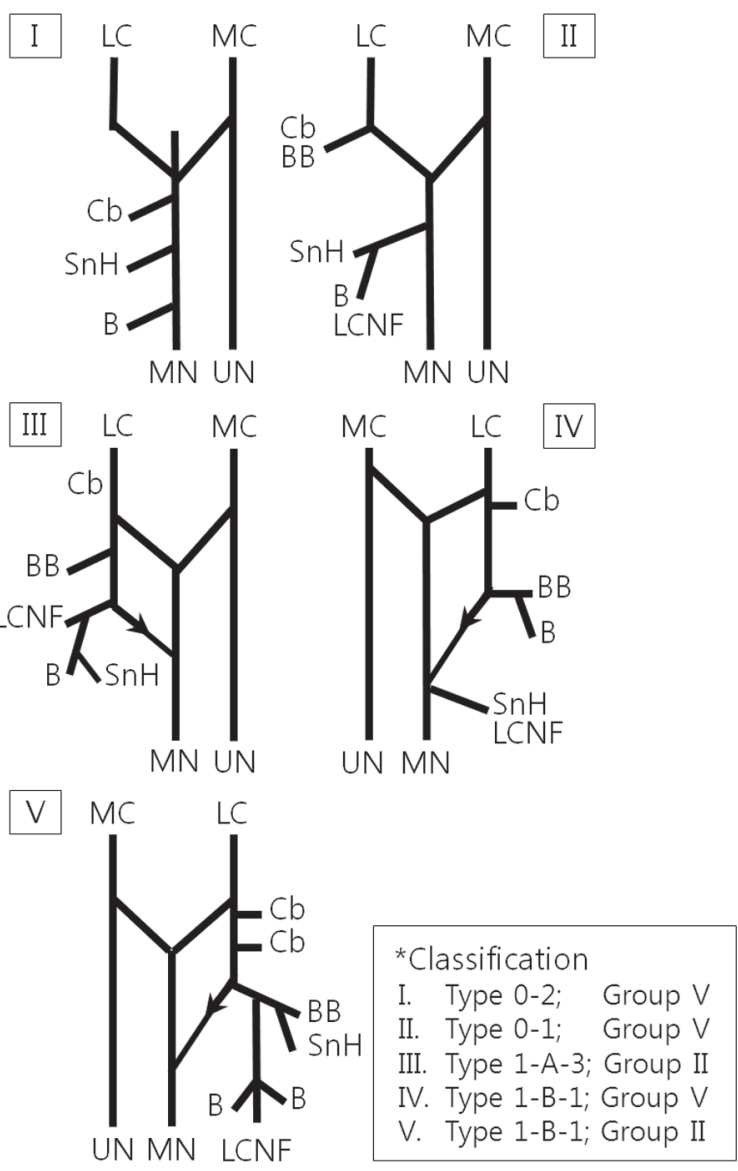

Figure 2. Schematic comparison of described in the literature on the combined neuromuscular variations of the supernumerary head of the biceps brachii muscle and a communicating branch of the musculocutaneous nerve; I. Arora and Dhingra (2005); II. Abu-Hijleh (2005); III. Pacholczak et al. (2011); IV. Abuel-Makarem et al. (2007); V. Present case. Each case was classified by Guerri-Guttenberg (2009; Type) and Kosugi et al. (1992; Group); LC — lateral cord; MC — medial cord; UN — ulnar nerve; MN — median nerve; $\mathrm{Cb}$ - nerve to coracobrachialis; $\mathrm{BB}$ - nerve to biceps brachii; $\mathrm{B}$ - nerve to brachialis; LCNF - lateral cutaneous nerve of forearm; $\mathrm{SnH}$ - nerve to supernumerary head of the biceps brachii.

the classification of Kosugi et al. [7], the present case and the case reported by Pacholczak et al. [10] correspond to Group II, while others correspond to Group $\mathrm{V}$. The present case was similar to those previously reported by Abuel-Makarem et al. [1] or Pacholczak et al. [10] but also has slightly different features (Fig. 2): 1) 2 muscular branches to the $\mathrm{Cb}, 2$ ) muscular branch to the supernumerary head of the BBM was derived from the branch to the BBM, and 3) the lateral cutaneous nerve of forearm was accompanied with 2 muscular branches to the brachialis muscle.

The development of the upper limb may explain the combined anatomical variations. When mesen- chyme migrates and forms the muscles into the limb bud, the mesenchyme is penetrated by the ventral primary rami of the appropriate spinal nerves. When some signalling between mesenchymal cells and the neuronal growth cones at the time of the fission of brachial plexus were altered, the altered signalling might result in variations of the neuromuscular system of the upper limb. Disturbances in these processes, taking place in the $4^{\text {th }} 7^{\text {th }}$ weeks of development, lead to anatomical variations in the innervation of muscles by appropriate nerves [11]. The MN is formed by a combination of ventral segmental branches and the $\mathrm{McN}$ arises from it [11], which means that existence of the communicating branch from the McN to the $\mathrm{MN}$, including this case, can be an expected type of variations. The additional head of the BBM is known to be derived from brachialis muscle embryologically [12]. During embryologic development, the brachialis muscle had some portion of which the distal insertion translocated from the ulna to the radius and became the supernumerary head of the BBM. Because the brachialis muscle is innervated by the McN, the supernumerary head of the BBM is also innervated by the $\mathrm{McN}$ as appeared in the present case also.

The relationship between the anomalous course of the $\mathrm{McN}$ and the occurrence of the additional head of the BBM has been previously discussed after the report of Ferner [4]. It is well known that mechanism of the formation of the variation of the $\mathrm{McN}$ fused with the MN was caused by the lack of the superficial head of the $\mathrm{Cb}$ separating both nerves. Therefore, the variation of the $\mathrm{McN}$ and the BBM should be considered as independent. Nevertheless, the presence of a supernumerary head of the BBM seemed to affect the course and branching of the $\mathrm{McN}$. It was reinforced by the fact that the frequency of communication between the $\mathrm{McN}$ and the MN was $21.8 \%$ with normal BBM [8], while it was $54.7 \%$ with a supernumerary head of the BBM [7]. To our knowledge, the combined variations with an unusual branching and course of the $\mathrm{McN}$ and a supernumerary head of the BBM in the present case have never been reported in previous papers. Lack of awareness of variations with communications between the $\mathrm{McN}$ and the MN might complicate surgical repair of the nerves when there were the excessive heads of the BBM especially. Knowing different patterns of the $\mathrm{McN}$ variation associated with the BBM variation is essential to prevent a surgeon from confusing clinically. 


\section{ACKNOWLEDGEMENTS}

This research was supported by the 2014 scientific promotion program funded by Jeju National University.

\section{REFERENCES}

1. Abuel-Makarem SM, Ibrahim AF, Darwish HH (2007) Absence of musculocutaenous nerve associated with a third head of biceps brachii muscle and entrapment of ulnar nerve. Neurosciences (Riyadh), 12: 340-342.

2. Abu-Hijleh MF (2005) Three-headed biceps brachii muscle associated with ducplicated musculocutaneous nerve. Clin Anat, 18: 376-379.

3. Arora L, Dhingra R (2005) Absence of musculocutaneous nerve and accessory head of biceps brachii: a case report. Indian J Plast Surg, 38: 144-146.

4. Ferner H (1938) Der Nervus musculocutaneous, seine Verlaufsvarietaten am Oberam und deren Beziehungen zur Entwicklung eines Caput tertium bicipitis. Z Anat Entwicklungsgesch, 108: 567-586.

5. Guerri-Guttenberg RA, Ingolotti M (2009) Classifying musculocutaneous nerve variations. Clin Anat, 22: 671-683.

6. Johnson E, Vekris M, Demesticha T, Soucacos P (2010) Neuroanatomy of the brachial plexus: normal and va- riant anatomy of its formation. Surg Radiol Anat, 32: 291-297.

7. Kosugi K, Shibata S, Yamashita H (1992) Supernumerary head of biceps brachii and branching pattern of the musculocutaneous nerve in Japanese. Surg Radiol Anat, 14: 175-185.

8. Kosugi K, Morita T, Koda M, Yamashita H (1986) Branching pattern of musculocutaneous nerve 1. Cases possessing normal biceps brachii muscle. Jikeikai Med J, 33: 63-71.

9. Maeda S, Katsushi K, Koizumi M, Ide J, Tokiyoski A, Mizuta H, Kodama K (2009) Morphological study of the communication between the musculocutaneous and median nerves. Anat Sci Int, 84: 34-40.

10. Pacholczak R, Klimek-Piotrowska W, Walocha JA (2011) Absence of the musculocutaneous nerve associated with a supernumerary head of biceps brachii: a case report. Surg Radiol Aant, 33: 551-554.

11. Prasada Rao PV, Chaudhary SC (2000) Communication of the musculocutaneous nerve with the median nerve. East Afr Med J, 77: 498-503.

12. Rai R, Ranade AV, Prabhu LV, Pai MM, Prakash (2007) Third head of biceps brachii in an Indian population. Singapore Med J, 48: 929-931.

13. Rodriguez-Niedenfuhr M, Vazquez T, Choi D, Parkin I, Sanudo JR (2003) Supernumerary humeral heads of the biceps brachii muscle revisited. Clin Anat, 16: 197-203. 